\title{
Bounds on the interior geometry and pressure profile of static fluid spheres
}

\author{
Damien Martin, Matt Visser \\ School of Mathematical and Computing Sciences, Victoria University of Wellington, \\ PO Box 600, Wellington, New Zealand \\ E-mail: matt.visser@vuw.ac.nz
}

\begin{abstract}
It is a famous result of relativistic stellar structure that (under mild technical conditions) a static fluid sphere satisfies the Buchdahl-Bondi bound $2 M / R \leq$ $8 / 9$; the surprise here being that the bound is not $2 M / R \leq 1$. In this article we provide further generalizations of this bound by placing a number of constraints on the interior geometry (the metric components), on the local acceleration due to gravity, on various combinations of the internal density and pressure profiles, and on the internal compactness $2 m(r) / r$ of static fluid spheres. We do this by adapting the standard tool of comparing the generic fluid sphere with a Schwarzschild interior geometry of the same mass and radius - in particular we obtain several results for the pressure profile (not merely the central pressure) that are considerably more subtle than might first be expected.
\end{abstract}

Dated: 10 June 2003; $\mathrm{LAT}_{\mathrm{E} X} \mathrm{X}$-ed 17 November 2018

PACS numbers: gr-qc/0306038 


\section{Introduction}

A useful pedagogical tool in teaching general relativity is the use of simple modelindependent bounds on stellar structure, or more generally on compact fluid spheres. In the Newtonian case simple bounds are nicely summarized by Chandrasekhar, in his book "Stellar structure" [1], while in general relativity the most famous of the modelindependent bounds is the Buchdahl-Bondi limit $[2,3]$ whereby the total mass and radius of any fluid body is bounded by

$$
\frac{2 M}{R}<\frac{8}{9}
$$

Note that this is stronger than the obvious bound $2 M / R<1$ that would most naively be expected from the absence of a black hole event horizon.

Now the Buchdahl-Bondi limit is discussed in many of the standard textbooks (such as Wald "General Relativity" [4], or Weinberg "Gravitation and Cosmology" [5]) but the discussion can be greatly enhanced to place limits not only on the global compactness $[\equiv 2 M / R]$, but also on additional features such as:

- the "internal compactness" $[\equiv 2 m(r) / r]$,

- the locally measured acceleration due to gravity,

- the metric coefficients of the spacetime,

- various linear combinations of the pressure and density profiles,

at all points throughout the star. The discussion below considerably extends what we have been able to find in the literature, but because of its elementary nature is particularly useful when teaching a first course in general relativity.

Key novel results are a number of bounds on the pressure profile $p(r)$. These include a relativistic extension of Chandrasekhar's bound, notable for its simplicity, and a number of constraints relating the pressure profile to that of the Schwarzschild interior geometry. We also discuss the central redshift — a quantity which is much less commonly discussed than the surface redshift. While the surface redshift can be measured directly by looking at optical spectral lines determining the central redshift would require, for instance, good spectral information (and theoretical modelling) regarding neutrino fluxes from the core. Though not a simple task, one of the payoffs would lie in thereby extracting information regarding the central pressure from the central redshift - an inequality on $z_{c}$ for general fluid spheres will be one of the results of this analysis. As always, bounds of this type are a trade-off between:

- the ease which with the bound can be stated;

- the ease which with the bound can be proved;

- the ease which with the bound can be used;

- the generality of the bound; and,

- the strength of the bound.

We have sought a compromise that yields useful physical information for physically reasonable fluid spheres. 


\section{Newtonian Stars}

For a Newtonian star the key structure equation is the equation of internal equilibrium [we adopt units where $G \equiv 1$ ]

$$
\frac{\mathrm{d} p}{\mathrm{~d} r}=-\frac{\rho(r) m(r)}{r^{2}}=-\frac{m^{\prime}(r) m(r)}{4 \pi r^{4}} .
$$

Chandrasekhar [1], following on and adapting earlier classical work, uses this to establish several theorems of which one stands out as the most important for the present purposes:

Theorem 1 Provided the average density $\bar{\rho}(r)=m(r) /\left[(4 \pi / 3) r^{3}\right]$ is nonincreasing as one moves outward in the star, then so is the quantity

$$
p(r)+\frac{3}{8 \pi} \frac{m(r)^{2}}{r^{4}} \text {. }
$$

Proof:

$$
\begin{aligned}
\frac{\mathrm{d}}{\mathrm{d} r}\left[p(r)+\frac{3}{8 \pi} \frac{m(r)^{2}}{r^{4}}\right] & =\frac{\mathrm{d} p}{\mathrm{~d} r}+\frac{3}{4 \pi} \frac{m(r) m^{\prime}(r)}{r^{4}}-\frac{3}{2 \pi} \frac{m(r)^{2}}{r^{5}} \\
& =\frac{1}{2 \pi} \frac{m(r)}{r^{4}}\left[m^{\prime}(r)-\frac{3 m(r)}{r}\right] \\
& =\frac{1}{2 \pi} \frac{m(r)}{r} \frac{\mathrm{d}}{\mathrm{d} r}\left[\frac{m(r)}{r^{3}}\right] \\
& =\frac{2}{3} \frac{m(r)}{r} \frac{\mathrm{d}}{\mathrm{d} r}[\bar{\rho}(r)] \\
& \leq 0 .
\end{aligned}
$$

Corollary 1 In a Newtonian star where the average density is nonincreasing outwards

$$
p_{c} \geq p(r)+\frac{3}{8 \pi} \frac{m(r)^{2}}{r^{4}} \geq \frac{3}{8 \pi} \frac{M^{2}}{R^{4}} .
$$

The net result of this theorem is to give robust and useful bounds on the pressure and density distribution inside a Newtonian star with only minimal input information. We will seek similar robust and simple results for general relativistic stars.

\section{TOV Stars}

The key structure equation for a relativistic star is the Tolman-Oppenheimer-Volkoff [TOV] equation

$$
\frac{\mathrm{d} p(r)}{\mathrm{d} r}=-\frac{[\rho(r)+p(r)]\left[m(r)+4 \pi p(r) r^{3}\right]}{r^{2}[1-2 m(r) / r]} .
$$

The most obvious point to make is that because density, pressure, and mass are all assumed positive in any reasonable star we now have the inequality

$$
\frac{\mathrm{d} p(r)}{\mathrm{d} r} \leq-\frac{\rho(r) m(r)}{r^{2}}=-\frac{m^{\prime}(r) m(r)}{4 \pi r^{4}} .
$$

Consequently the Newtonian theorem and its corollary still holds in a relativistic star

- the only modification is in the proof of the theorem; where the equality on going 
from line 1 to line 2 is now an inequality. Of course this is a very weak-constraint on the structure of a relativistic star, and much better constraints are available. A particularly simple constraint is based on the fact that for a relativistic star the positivity of pressure implies

$$
\frac{\mathrm{d} p(r)}{\mathrm{d} r} \leq-\frac{\rho(r) m(r)}{r^{2}[1-2 m(r) / r]}=-\frac{m^{\prime}(r) m(r)}{4 \pi r^{4}[1-2 m(r) / r]}
$$

Note that by dropping both linear and quadratic pressure terms on the RHS we are implicitly ignoring the "regeneration of pressure" [6]; consequently the bound we derive will be relatively weak but has the advantage of an elementary proof.

Theorem 2 Provided the average density $\bar{\rho}(r)=m(r) /\left[(4 \pi / 3) r^{3}\right]$ is nonincreasing as one moves outward in a relativistic star, then so is the quantity

$$
p(r)-\frac{3}{16 \pi} \frac{m(r)}{r^{3}} \ln \left[1-\frac{2 m(r)}{r}\right] \text {. }
$$

Proof:

$$
\begin{aligned}
\frac{\mathrm{d}}{\mathrm{d} r}\{p(r) & \left.-\frac{3}{16 \pi} \frac{m(r)}{r^{3}} \ln \left[1-\frac{2 m(r)}{r}\right]\right\} \\
& =\frac{\mathrm{d} p}{\mathrm{~d} r}-\frac{3}{16 \pi} \frac{\mathrm{d}}{\mathrm{d} r}\left[\frac{m(r)}{r^{3}}\right] \ln \left[1-\frac{2 m(r)}{r}\right] \\
& \quad+\frac{3}{16 \pi} \frac{m(r)}{r^{3}} \frac{1}{1-2 m(r) / r} \frac{\mathrm{d}}{\mathrm{d} r}\left[\frac{2 m(r)}{r}\right] \\
& \leq \frac{1}{8 \pi} \frac{m(r)}{r^{4}} \frac{1}{1-2 m(r) / r}\left[m^{\prime}(r)-\frac{3 m(r)}{r}\right] \\
& =\frac{1}{8 \pi} \frac{m(r)}{r} \frac{1}{1-2 m(r) / r} \frac{\mathrm{d}}{\mathrm{d} r}\left[\frac{m(r)}{r^{3}}\right] \\
& \leq 0 .
\end{aligned}
$$

Corollary 2 In a relativistic star, provided the average density is nonincreasing as one moves outward

$$
p_{c} \geq p(r)-\frac{3}{16 \pi} \frac{m(r)}{r^{3}} \ln \left[1-\frac{2 m(r)}{r}\right] \geq-\frac{3}{16 \pi} \frac{M}{R^{3}} \ln \left[1-\frac{2 M}{R}\right] .
$$

Comment:

Note that as $2 M / R \rightarrow 0$ one recovers the Newtonian result, and that as $2 M / R \rightarrow 1$ the central pressure is forced to infinity. This argument is not good enough to reproduce the Buchdahl-Bondi limit, but it is elementary, and it is sufficiently good to show the runaway explosion of central pressure for sufficiently compact objects. Moreover, because in deriving this bound we have not used the "regeneration of pressure" effect [6], it follows that the occurrence of unbounded central pressures in general relativity is not intrinsically a regeneration effect.

To now obtain the Buchdahl-Bondi bound, and its extensions, the best strategy is to develop a number of comparison theorems that provide inequalities relating the generic relativistic fluid sphere and the interior Schwarzschild solution. 
Bounds on the interior geometry and pressure profile of static fluid spheres

\section{Generic interior geometry}

Let us adopt coordinates to write the general interior geometry in the form

$$
\mathrm{d} s^{2}=-\zeta(r)^{2} \mathrm{~d} t^{2}+\frac{\mathrm{d} r^{2}}{1-2 m(r) / r}+r^{2}\left[\mathrm{~d} \theta^{2}+\sin ^{2} \theta \mathrm{d} \phi^{2}\right] .
$$

Equivalently

$$
\mathrm{d} s^{2}=-\exp \left[-2 \int_{r}^{\infty} g(\tilde{r}) \mathrm{d} \tilde{r}\right] \mathrm{d} t^{2}+\frac{\mathrm{d} r^{2}}{1-2 m(r) / r}+r^{2}\left[\mathrm{~d} \theta^{2}+\sin ^{2} \theta \mathrm{d} \phi^{2}\right] .
$$

With this choice $g(r)$ is positive for an inward gravitational attraction and equals the locally measured acceleration due to gravity. The Einstein equations yield

$$
\begin{aligned}
8 \pi \rho & =G_{\hat{t} \hat{t}}=2 m^{\prime}(r) / r^{2} ; \\
8 \pi p & =G_{\hat{r} \hat{r}}=2\left\{\frac{g(r)[1-2 m(r) / r]}{r}-\frac{m(r)}{r^{3}}\right\} ; \\
8 \pi p=G_{\hat{\theta} \hat{\theta}} & =-\frac{m^{\prime}(r)[1+r g(r)]}{r^{2}}+\frac{m(r)\left[1-r g(r)-2 r^{2} g^{\prime}(r)-2 r^{2} g(r)^{2}\right]}{r^{3}} \\
+ & \frac{g(r)[1+r g(r)]}{r} .
\end{aligned}
$$

The first of these integrates to

$$
m(r)=\int_{0}^{r} 4 \pi \rho(\tilde{r}) \tilde{r}^{2} \mathrm{~d} \tilde{r}
$$

which justifies the choices of notation $g_{r r}=1 /[1-2 m(r) / r]$. The second equation can be algebraically rearranged to yield

$$
g(r)=\frac{m(r)+4 \pi p(r) r^{3}}{r^{2}[1-2 m(r) / r]}
$$

The third equation is quite messy - however in view of the Bianchi identities it may be "traded off" for the covariant conservation of stress energy which [for isotropic pressures] takes the rather simple form

$$
p^{\prime}(r)=-[\rho(r)+p(r)] g(r)
$$

Eliminating $g(r)$ leads to the TOV equation as previously given.

If we choose to work in terms of $\zeta(r)$ instead of $g(r)$, and we shall soon see that this is sometimes useful, the only significant change in appearance [not substance] is in the equation

$$
G_{\hat{\theta} \hat{\theta}}=\frac{\zeta(r)^{\prime \prime}[1-2 m(r) / r]}{\zeta(r)}+\frac{\zeta^{\prime}(r)\left[1-m(r) / r-m^{\prime}(r)\right]}{r \zeta(r)}-\frac{[m(r) / r]^{\prime}}{r} .
$$

For the $G_{\hat{r} \hat{r}}$ equation there is very little change

$$
G_{\hat{r} \hat{r}}=2\left\{\frac{\zeta^{\prime}(r)}{\zeta(r)} \frac{[1-2 m(r) / r]}{r}-\frac{m(r)}{r^{3}}\right\} .
$$




\section{Interior Schwarzschild}

The interior Schwarzschild solution is a specific solution of the Einstein equations corresponding to a star whose density is constant throughout space. (Despite the common misperception, this does not mean the star is "incompressible", see Misner, Thorne, and Wheeler for details [7], see esp pp. 609 ff.) If we write the metric as

$$
\mathrm{d} s_{*}^{2}=-\zeta_{*}(r)^{2} \mathrm{~d} t^{2}+\frac{\mathrm{d} r^{2}}{1-2 m_{*}(r) / r}+r^{2}\left[\mathrm{~d} \theta^{2}+\sin ^{2} \theta \mathrm{d} \phi^{2}\right],
$$

then solving the Einstein equations for $\rho=\rho_{*}$ yields the standard textbook results [4, $5,7]$ :

$$
\begin{aligned}
& m_{*}(r)=\frac{4 \pi}{3} \rho_{*} r^{3}=M \frac{r^{3}}{R^{3}}, \\
& \zeta_{*}(r)=\frac{1}{2}\left[3 \sqrt{1-2 M / R}-\sqrt{1-2 m_{*}(r) / r}\right],
\end{aligned}
$$

and

$$
p_{*}(r)=\rho_{*} \frac{\sqrt{1-2 m_{*}(r) / r}-\sqrt{1-2 M / R}}{3 \sqrt{1-2 M / R}-\sqrt{1-2 m_{*}(r) / r}} .
$$

Here and henceforth a subscript or superscript star on a quantity refers to the Schwarzschild interior solution. The central pressure is

$$
p_{c}^{*}=\rho_{*} \frac{1-\sqrt{1-2 M / R}}{3 \sqrt{1-2 M / R}-1},
$$

which certainly diverges as $2 M / R \rightarrow 8 / 9$; but this is not yet enough to establish the Buchdahl-Bondi bound - to do so we need to develop theorems relating the general interior geometry (for an object with the same mass $M$ and radius $R$ ) to the specific Schwarzschild interior geometry. At the center of the interior Schwarzschild geometry we also have

$$
\zeta_{c}^{*}=\frac{1}{2}[3 \sqrt{1-2 M / R}-1]
$$

which is related to the central redshift by

$$
z_{c}^{*}=\frac{1}{\zeta_{c}^{*}}-1=\frac{3 p_{c}^{*}}{\rho_{*}} .
$$

As useful algebraic results note that

$$
\begin{aligned}
& p_{*}(r) \zeta_{*}(r)=\frac{\rho_{*}}{2}\left[\sqrt{1-2 m_{*}(r) / r}-\sqrt{1-2 M / R}\right], \\
& {\left[\rho_{*}+p_{*}(r)\right] \zeta_{*}(r)=\rho_{*} \sqrt{1-2 M / R},}
\end{aligned}
$$

and

$$
\left[\rho_{*}+3 p_{*}(r)\right] \zeta_{*}(r)=\rho_{*} \sqrt{1-2 m_{*}(r) / r} .
$$




\section{Comparison theorems for the spacetime geometry}

Now consider a generic self-gravitating relativistic fluid sphere

Lemma 1 If the average density $\bar{\rho}$ is nonincreasing outwards, then

$$
m(r) \geq m_{*}(r) \equiv M \frac{r^{3}}{R^{3}} .
$$

Proof:

By definition and hypothesis

$$
\bar{\rho}(r) \equiv \frac{3 m(r)}{4 \pi r^{3}} \geq \bar{\rho}(R) \equiv \frac{3 M}{4 \pi R^{3}} \equiv \rho_{*} .
$$

Lemma 2 The average density $\bar{\rho}$ is nonincreasing outwards if and only if for all $r$

$$
\bar{\rho}(r) \geq \rho(r) .
$$

Proof:

By definition and hypothesis

$$
\frac{\mathrm{d} \bar{\rho}(r)}{\mathrm{d} r}=\frac{\mathrm{d}}{\mathrm{d} r}\left[\frac{3 m(r)}{4 \pi r^{3}}\right]=\frac{3}{r}[\rho(r)-\bar{\rho}(r)] .
$$

Note that if the unaveraged density $\rho(r)$ is nonincreasing outwards then certainly $\bar{\rho} \geq \rho$ and so the average density $\bar{\rho}(r)$ is also nonincreasing outwards; the converse however does not hold. A nonincreasing average density does not necessarily imply a nonincreasing density and is a genuinely weaker constraint. From the first lemma it trivially follows that:

Lemma 3 If the average density $\bar{\rho}$ is nonincreasing outwards, then

$$
g_{r r}(r) \geq g_{r r}^{*}(r) .
$$

and

$$
m(r) / r \geq m_{*}(r) / r .
$$

Much more subtle is the following result regarding the $t t$ metric component:

Theorem 3 If the average density $\bar{\rho}$ is nonincreasing outwards, then

$$
\zeta(r) \leq \zeta_{*}(r)
$$

Equivalently

$$
\left|g_{t t}(r)\right| \leq\left|g_{t t}^{*}(r)\right|
$$

Proof:

Obtaining this result is somewhat tedious but relatively straightforward — an adaptation of the discussion in Weinberg [5] or Wald [4] suffices. With a little more work, you could also adapt the discussion in the original 1959 Buchdahl paper [2]. Consider

$$
\begin{aligned}
0 & =\left[G_{\hat{r} \hat{r}}-G_{\hat{\theta} \hat{\theta}}\right] r^{3} \zeta(r) \\
& =-r^{3} \zeta^{\prime \prime}(r)[1-2 m(r) / r]+\zeta^{\prime}(r)\left[r^{2}+r^{2} m^{\prime}(r)-3 r m(r)\right]+\zeta(r)\left[r m^{\prime}(r)-3 m(r)\right] .
\end{aligned}
$$


Rearranging

$$
\frac{\mathrm{d}}{\mathrm{d} r}\left[\frac{1}{r} \sqrt{1-\frac{2 m(r)}{r}} \zeta^{\prime}(r)\right]=\frac{\zeta(r)}{\sqrt{1-2 m(r) / r}} \frac{\mathrm{d}}{\mathrm{d} r}\left[\frac{m(r)}{r^{3}}\right] .
$$

So, as long as the average density is nonincreasing as we go outward

$$
\frac{\mathrm{d}}{\mathrm{d} r}\left[\frac{1}{r} \sqrt{1-\frac{2 m(r)}{r}} \zeta^{\prime}(r)\right] \leq 0
$$

At the surface of the star $\zeta(r)$ must match smoothly onto the exterior Schwarzschild geometry, therefore

$$
\zeta(R)=\sqrt{1-\frac{2 M}{R}} ; \quad \zeta^{\prime}(R)=\frac{M}{R^{2}} \frac{1}{\sqrt{1-2 M / R}} .
$$

Combining (50) with this boundary condition implies $[\forall r \in(0, R)]$

$$
\zeta^{\prime}(r) \geq \frac{M r}{R^{3}} \frac{1}{\sqrt{1-2 m(r) / r}}=\frac{m_{*}(r)}{r^{2}} \cdot \frac{1}{\sqrt{1-2 m(r) / r}}
$$

Integrate from $r$ to $R$, [not from 0 to $R$ ], we have

$$
\zeta(R)-\zeta(r) \geq \frac{M}{R^{3}} \int_{r}^{R} \frac{\tilde{r}}{\sqrt{1-2 m(\tilde{r}) / \tilde{r}}} \mathrm{~d} \tilde{r} .
$$

But in the RHS, $m(r) \geq m_{*}(r)$, so

$\zeta(R)-\zeta(r) \geq \frac{M}{R^{3}} \int_{r}^{R} \frac{\tilde{r}}{\sqrt{1-2 m_{*}(\tilde{r}) / \tilde{r}}} \mathrm{~d} \tilde{r}=\frac{M}{R^{3}} \int_{r}^{R} \frac{\tilde{r}}{\sqrt{1-2 M \tilde{r}^{2} / R^{3}}} \mathrm{~d} \tilde{r}$.

This integral is now do-able in closed form, with the result

$$
\zeta(R)-\zeta(r) \geq-\frac{1}{2}\left[\sqrt{1-\frac{2 M \tilde{r}^{2}}{R^{3}}}\right]_{r}^{R} .
$$

Rearranging, and applying the boundary condition at $R$, we have

$$
\zeta(r) \leq \frac{1}{2}\left[3 \sqrt{1-\frac{2 M}{R}}-\sqrt{1-\frac{2 M r^{2}}{R^{3}}}\right] .
$$

The RHS is now recognizable as $\zeta_{*}(r)$, the quantity appropriate to the Schwarzschild interior solution, so the theorem is proved.

\section{Comment:}

- Note what has happened here: Both of the [physically nontrivial] metric components, $g_{t t}$ and $g_{r r}$, have been bounded in terms of what their values would have been for a Schwarzschild interior solution of the same mass and radius. This now is more than enough to deduce the Buchdahl-Bondi bound. Since $\zeta(r) \leq \zeta_{*}(r)$, and since $\zeta_{*}(r) \rightarrow 0$ for some finite positive $r$ whenever $2 M / R>8 / 9$, we deduce that any star [in which the average density is nonincreasing outwards] must likewise satisfy the same bound. More formally: 
Corollary 3 If the average density $\bar{\rho}$ is nonincreasing outwards, then

$$
\frac{2 M}{R} \leq \frac{8}{9}
$$

Proof:

If the center of the star is to be regular then we must have

$$
0 \leq \zeta_{c} \leq \zeta_{c}^{*}=\frac{1}{2}\left[3 \sqrt{1-\frac{2 M}{R}}-1\right]
$$

Comment:

- It might be tempting to conclude that for all $r$

$$
\text { i.i. } \frac{2 m(r)}{r} \leq \frac{8}{9} \quad \text { ??? }
$$

Although true, this conclusion cannot be drawn from the arguments so far presented.

- Note also what has not happened here. We cannot [at this stage] deduce any bound on the pressures. Indeed it might be tempting to assert

$$
\text { i.i. } \quad p(r) \geq p_{*}(r) \quad \text { ??? }
$$

but such an assertion would actually be false. To see this compare

$$
\left.\frac{\mathrm{d} p}{\mathrm{~d} r}\right|_{R}=\rho(R) \frac{M}{R^{2}} \frac{1}{1-2 M / R} ;
$$

and

$$
\left.\frac{\mathrm{d} p_{*}}{\mathrm{~d} r}\right|_{R}=\rho_{*} \frac{M}{R^{2}} \frac{1}{1-2 M / R} \geq\left.\frac{\mathrm{d} p}{\mathrm{~d} r}\right|_{R} .
$$

Thus sufficiently near the surface we must actually have $p(r) \leq p_{*}(r)$; though at the center (as we shall soon see) $p(0) \geq p_{*}(0)$. Thus general bounds on the central pressure and pressure profile are trickier to establish (which is why, regarding the central pressure, Weinberg resorts to "it can be shown that", and Wald remains silent).

As well as the metric components, there is a similar constraint on the locally measured acceleration due to gravity detected by static observers.

Lemma 4 If the average density $\bar{\rho}$ is nonincreasing outwards, then

$$
g(r) \geq g^{*}(r) .
$$

Proof:

To see this use the bound (52) to obtain

$$
\zeta^{\prime}(r) \geq \frac{m_{*}(r)}{r^{2}} \frac{1}{\sqrt{1-2 m(r) / r}} \geq \frac{m_{*}(r)}{r^{2}} \frac{1}{\sqrt{1-2 m_{*}(r) / r}}=\zeta_{*}^{\prime}(r),
$$

and then combine this, the definition of $g$, and (46), to obtain

$$
g(r)=\frac{\zeta^{\prime}(r)}{\zeta(r)} \geq \frac{\zeta_{*}^{\prime}(r)}{\zeta_{*}(r)}=g^{*}(r) .
$$


Bounds on the interior geometry and pressure profile of static fluid spheres

7. Bounds on the internal compactness $2 m(r) / r$

Theorem 4 If the average density $\bar{\rho}$ is nonincreasing outwards, then

$$
\frac{2 m(r)}{r} \leq \frac{8}{9}\left[1+\frac{\sqrt{1+6 \pi p(r) r^{2}}}{2}-\frac{1+6 \pi p(r) r^{2}}{2}\right]
$$

Proof:

This particular result is derived, for instance, in Wald, "General Relativity" [4]. To obtain a bound on $2 m(r) / r$ modify the preceding argument by now considering the region $\tilde{r} \in(0, r)$ with $r<R$. Then, following the discussion in Wald or using equation (50) above we have for $\tilde{r} \leq r$

$$
\frac{1}{\tilde{r}} \sqrt{1-\frac{2 m(\tilde{r})}{\tilde{r}}} \zeta^{\prime}(\tilde{r}) \geq \frac{1}{r} \sqrt{1-\frac{2 m(r)}{r}} \zeta^{\prime}(r) .
$$

The RHS evaluates to

$\frac{1}{r} \sqrt{1-\frac{2 m(r)}{r}} g(r) \zeta(r)=\frac{m(r)+4 \pi p(r) r^{3}}{r^{3} \sqrt{1-2 m(r) / r}} \zeta(r)=\frac{4 \pi p(r)+m(r) / r^{3}}{\sqrt{1-2 m(r) / r}} \zeta(r)$.

Thus

$$
\zeta^{\prime}(\tilde{r}) \geq \zeta(r) \frac{4 \pi p(r)+m(r) / r^{3}}{\sqrt{1-2 m(r) / r}} \frac{\tilde{r}}{\sqrt{1-2 m(\tilde{r}) / \tilde{r}}} .
$$

It is now sufficient to integrate $\tilde{r}$ from zero to $r$ :

$$
\zeta(r)-\zeta(0) \geq \zeta(r) \frac{4 \pi p(r)+m(r) / r^{3}}{\sqrt{1-2 m(r) / r}} \int_{0}^{r} \frac{\tilde{r}}{\sqrt{1-2 m(\tilde{r}) / \tilde{r}}} \mathrm{~d} \tilde{r} .
$$

That is

$$
\zeta(0) \leq \zeta(r)\left\{1-\frac{4 \pi p(r)+m(r) / r^{3}}{\sqrt{1-2 m(r) / r}} \int_{0}^{r} \frac{\tilde{r}}{\sqrt{1-2 m(\tilde{r}) / \tilde{r}}} \mathrm{~d} \tilde{r} .\right\}
$$

But because $\zeta(0)$ must be positive in a star whose geometry remains regular all the way to the center, the quantity in braces must be positive, and [independent of the unknown value of $\zeta(r)$ ] we have the inequality

$$
\sqrt{1-\frac{2 m(r)}{r}} \geq\left[4 \pi p(r)+\frac{m(r)}{r^{3}}\right] \int_{0}^{r} \frac{\tilde{r}}{\sqrt{1-2 m(\tilde{r}) / \tilde{r}}} \mathrm{~d} \tilde{r} .
$$

But, because the average density is nonincreasing outwards, our first lemma implies

$$
m(\tilde{r}) \geq m(r) \frac{\tilde{r}^{3}}{r^{3}}
$$

This implies

$$
\sqrt{1-\frac{2 m(r)}{r}} \geq\left[4 \pi p(r)+\frac{m(r)}{r^{3}}\right] \int_{0}^{r} \frac{\tilde{r}}{\sqrt{1-2 m(r) \tilde{r}^{2} / r^{3}}} \mathrm{~d} \tilde{r} .
$$

The integral is now elementary, with the result

$$
\sqrt{1-\frac{2 m(r)}{r}} \geq\left[4 \pi p(r)+\frac{m(r)}{r^{3}}\right] \frac{r^{3}}{2 m(r)}\left\{1-\sqrt{1-\frac{2 m(r)}{r}}\right\} .
$$


Consequently

$$
\frac{2 m(r)}{r} \sqrt{1-\frac{2 m(r)}{r}} \geq\left[4 \pi p(r) r^{2}+\frac{m(r)}{r}\right]\left\{1-\sqrt{1-\frac{2 m(r)}{r}}\right\} .
$$

Equivalently

$$
1-\frac{2 m(r)}{r}+\sqrt{1-\frac{2 m(r)}{r}} \geq 4 \pi p(r) r^{2}+\frac{m(r)}{r} .
$$

It is now a matter of tedious but straightforward algebra to rearrange this inequality into the desired form. Finally, because for positive $x$ one has $\sqrt{1+x}<1+x$ we deduce:

Corollary 4 If the average density $\bar{\rho}$ is nonincreasing outwards, then

$$
\frac{2 m_{*}(r)}{r} \leq \frac{2 m(r)}{r} \leq \frac{8}{9}
$$

Proof:

Simply use the fact that $p(r)$ is positive inside the star, and the previously derived lower bound on the compactness. If you prefer you can write this as

$$
2 M \frac{r^{2}}{R^{3}} \leq \frac{2 m(r)}{r} \leq \frac{8}{9} .
$$

Comment:

Since $2 m(r) / r$ is zero at the center, and equals $2 M / R$ at the surface it is tempting to suppose that $2 m(r) / r$ might be monotone increasing. In general it is not, and many equations of state are known for which $2 m(r) / r$ develops damped oscillatory behaviour as a function of $r[6,8]$.

Corollary 5 If the average density $\bar{\rho}$ is nonincreasing outwards, then

$$
p(r) \leq \frac{1}{4 \pi r^{2}}\left\{1-\frac{3 m(r)}{r}+\sqrt{1-\frac{2 m(r)}{r}}\right\} .
$$

Comment:

This bound is somewhat unusual in that it provides an upper bound on the pressure. It is less useful than one might imagine since it gives no information about the central pressure.

\section{Comparison theorems for the pressure profile}

General and stringent theorems regarding the central pressure and pressure profile [rather than metric coefficients and compactness] are relatively more cumbersome.

With the tools we have at hand, an easy result is this:

Theorem 5 If the average density $\bar{\rho}$ is nonincreasing outwards, then

$$
\frac{[\bar{\rho}(r)+3 p(r)] \zeta(r)}{\sqrt{1-2 m(r) / r}} \geq \rho_{*}=\frac{\left[\rho_{*}+3 p_{*}(r)\right] \zeta_{*}(r)}{\sqrt{1-2 m_{*}(r) / r}} .
$$


Proof:

First, by the geometric comparison theorems of the previous section, in particular equation (52),

$g(r)=\frac{\zeta^{\prime}(r)}{\zeta(r)} \geq \frac{M r}{R^{3}} \frac{1}{\sqrt{1-2 m(r) / r} \zeta(r)}=\frac{4 \pi \rho_{*} r}{3} \frac{1}{\sqrt{1-2 m(r) / r} \zeta(r)}$.

Second, by the $\hat{r} \hat{r}$ Einstein equation

$$
p=\frac{1}{4 \pi}\left\{\frac{g(r)[1-2 m(r) / r]}{r}-\frac{m}{r^{3}}\right\}=\frac{1}{4 \pi} \frac{g(r)[1-2 m(r) / r]}{r}-\frac{\bar{\rho}(r)}{3} .
$$

Combine. We have

$$
\bar{\rho}+3 p \geq \frac{\rho_{*} \sqrt{1-2 m(r) / r}}{\zeta(r)} .
$$

For the last equality use the properties of the interior Schwarzschild solution, as embodied in equation (39).

Corollary 6 If the average density $\bar{\rho}$ is nonincreasing outwards, then

$$
\zeta_{c} \geq \frac{\rho_{*}}{\rho_{c}+3 p_{c}} .
$$

For the central redshift

$$
z_{c} \leq \frac{\rho_{c}+3 p_{c}-\rho_{*}}{\rho_{*}} .
$$

Corollary 7 If the average density $\bar{\rho}$ is nonincreasing outwards, then

$$
\frac{\bar{\rho}(r)+3 p(r)}{\sqrt{1-2 m(r) / r}} \geq \frac{\rho_{*}}{\zeta_{*}(r)}=\frac{\rho_{*}+3 p_{*}(r)}{\sqrt{1-2 m_{*}(r) / r}} .
$$

Proof:

Apply the inequality $\zeta(r) \leq \zeta_{*}(r)$ to the preceding theorem.

Corollary 8 If the average density $\bar{\rho}$ is nonincreasing outwards, then

$$
\rho_{c}+3 p_{c} \geq \rho_{*}+3 p_{c}^{*} \text {. }
$$

Comment:

While somewhat crude, this bound has the benefit of being both elementary and guaranteeing that the central value of $\rho+3 p$ diverges at or before one reaches the Buchdahl-Bondi limit. Note that this does not permit us to place a bound on $p_{c}$ itself, only on the combination $\rho_{c}+3 p_{c}$.

The first bound we place on the pressure profile itself is this:

Theorem 6 If the average density $\bar{\rho}$ is nonincreasing outwards, then

$$
p(r) \geq \frac{\rho_{*}}{3} \frac{2 \sqrt{1-2 m(r) / r}+\sqrt{1-2 M r^{2} / R^{3}}-3 \sqrt{1-2 M / R}}{3 \sqrt{1-2 M / R}-\sqrt{1-2 M r^{2} / R^{3}}} .
$$


Proof:

Start from the equation $p^{\prime}=-[\rho+p] g=-[\rho+p] \zeta^{\prime} / \zeta$ and re-write it in the form

$$
[p \zeta]^{\prime}=-\rho \zeta^{\prime} .
$$

Integrate from $r$ to $R$, then

$$
p(r) \zeta(r)=\int_{r}^{R} \rho(\tilde{r}) \zeta^{\prime}(\tilde{r}) \mathrm{d} \tilde{r} .
$$

But because of our earlier inequality on $\zeta^{\prime}(r)$, embodied in equation (52),

$$
\begin{aligned}
& p(r) \zeta(r) \geq \int_{r}^{R} \rho(\tilde{r}) \frac{M \tilde{r}}{R^{3}} \frac{1}{\sqrt{1-2 m(\tilde{r}) / \tilde{r}}} \mathrm{~d} \tilde{r} \\
& =\frac{M}{R^{3}} \int_{r}^{R} \frac{m^{\prime}(\tilde{r})}{4 \pi \tilde{r} \sqrt{1-2 m(\tilde{r}) / \tilde{r}}} \mathrm{~d} \tilde{r} \\
& =\frac{\rho_{*}}{3} \int_{r}^{R}\left\{\left[\frac{m(\tilde{r})}{\tilde{r}}\right]^{\prime}+\frac{m(\tilde{r})}{\tilde{r}^{2}}\right\} \frac{1}{\sqrt{1-2 m(\tilde{r}) / \tilde{r}}} \mathrm{~d} \tilde{r} \\
& =\frac{\rho_{*}}{3}\left\{-\left.\sqrt{1-2 m(\tilde{r}) / \tilde{r}}\right|_{r} ^{R}+\int_{r}^{R} \frac{m(\tilde{r}) / \tilde{r}^{2}}{\sqrt{1-2 m(\tilde{r}) / \tilde{r}}} \mathrm{~d} \tilde{r}\right\} .
\end{aligned}
$$

Again using the fact that the average density is nonincreasing outwards

$$
p(r) \zeta(r) \geq \frac{\rho_{*}}{3}\left\{-\left.\sqrt{1-2 m(\tilde{r}) / \tilde{r}}\right|_{r} ^{R}+\int_{r}^{R} \frac{M \tilde{r} / R^{3}}{\sqrt{1-2 M \tilde{r}^{2} / R^{3}}} \mathrm{~d} \tilde{r}\right\} .
$$

The remaining integral is again elementary, with the consequence

$$
\begin{gathered}
p(r) \zeta(r) \geq \frac{\rho_{*}}{6}\left\{-2 \sqrt{1-2 M / R}+2 \sqrt{1-2 m(r) / r}-\sqrt{1-2 M / R}+\sqrt{1-2 M r^{2} / R^{3}}\right\} \\
=\frac{\rho_{*}}{6}\left\{2 \sqrt{1-2 m(r) / r}+\sqrt{1-2 M r^{2} / R^{3}}-3 \sqrt{1-2 M / R}\right\} .
\end{gathered}
$$

Finally, since we have already established $\zeta(r) \leq \zeta_{*}(r)$, inserting the explicit form of $\zeta_{*}(r)$ completes the proof.

Corollary 9 If the average density $\bar{\rho}$ is nonincreasing outwards, then

$$
\zeta(r) p(r)-\frac{\rho_{*}}{3} \sqrt{1-2 m(r) / r} \geq \zeta_{*}(r) p_{*}(r)-\frac{\rho_{*}}{3} \sqrt{1-2 m_{*}(r) / r}
$$

Proof:

This is a slight strengthening of the previous theorem obtained by not invoking $\zeta(r) \leq \zeta_{*}(r)$ at the last step. In this version of the result everything on the LHS refers to the body we are investigating and everything on the RHS refers to the interior Schwarzschild geometry we are using to compare it to.

Corollary 10 If the average density $\bar{\rho}$ is nonincreasing outwards, then

$$
p(r) \geq p_{*}(r)+\frac{\rho_{*}}{3}\left[\frac{\sqrt{1-2 m(r) / r}-\sqrt{1-2 m_{*}(r) / r}}{\zeta_{*}(r)}\right] .
$$


Proof:

This is simply a re-writing of the previous theorem to make it clear that the generic pressure bound consists of the interior Schwarzschild result plus a "correction" term. The correction term vanishes at both $R$ and at 0 , and is negative between these locations. Note that the bound is sharp in the sense that the interior Schwarzschild solution saturates the bound.

Comment:

Note that to bound the pressure profile $p(r)$ you need information regarding the local compactness $m(r) / r$, which is difficult to measure observationally. This contrasts with most of the other bounds derived in this article which depend on parameters that can be measured from the stellar exterior.

Comment:

If we take this pressure bound and consider it near the surface of the star then we find

$$
p(R) \geq 0,
$$

and

$$
\left.\frac{\mathrm{d} p}{\mathrm{~d} r}\right|_{R} \geq-\rho(R) \frac{M}{R^{2}(1-2 M / R)} .
$$

But, because of the boundary conditions at the surface of the star, these bounds are actually saturated by any fluid sphere, and cannot be improved.

Corollary 11 If the average density $\bar{\rho}$ is nonincreasing outwards, then

$$
p_{c} \geq p_{c}^{*} \text {. }
$$

Comment:

Thus the interior Schwarzschild solution really does provide a lower bound on the central density even if it cannot be used [in "uncorrected" form] elsewhere within the star. Consequently, in the Buchdahl-Bondi limit $2 M / R \rightarrow 8 / 9$ not only does $\zeta_{c} \rightarrow 0$, but we also have $p_{c} \rightarrow \infty$ [as intuitively expected].

We now consider several additional bounds that are derived in slightly different fashion.

Theorem 7 If the density $\rho$ [not the average density] is nonincreasing outwards, then in terms of the surface density $\rho_{s}$ :

$$
p(r)+\rho(r) \geq \rho_{s} \frac{\sqrt{1-2 M / R}}{\zeta(r)} \geq \rho_{s} \frac{\sqrt{1-2 M / R}}{\zeta_{*}(r)} .
$$

Proof:

Start from the equation $p^{\prime}=-[\rho+p] g=-[\rho+p] \zeta^{\prime} / \zeta$ and re-write it in the form

$$
[(p+\rho) \zeta]^{\prime}=+\rho^{\prime} \zeta \leq 0
$$

Integrate from $r$ to $R$, then

$$
\rho_{s} \sqrt{1-2 M / R}-[p(r)+\rho(r)] \zeta(r) \leq 0 .
$$

Rearrange, and use the previous inequality for $\zeta(r)$. 


\section{Comment:}

This is a relatively weak bound because it involves the surface density $\rho_{s}$. On the other hand, the RHS of the bound is now expressed in terms of quantities that are in principle accessible via external observation.

Corollary 12 If the density $\rho$ is nonincreasing outwards, then

$$
p_{c}+\rho_{c} \geq \rho_{s} \frac{\sqrt{1-2 M / R}}{\zeta_{c}^{*}}=\rho_{s} \frac{2 \sqrt{1-2 M / R}}{3 \sqrt{1-2 M / R}-1} .
$$

The theorem above can be considerably strengthened by noting that $p^{\prime}=-[\rho+p] g=$ $-[\rho+p] \zeta^{\prime} / \zeta$ implies the exact general result

$$
\zeta(r)=\zeta_{c} \exp \left[-\int_{0}^{r} \frac{\mathrm{d} p / \mathrm{d} r}{\rho(\tilde{r})+p(\tilde{r})} \mathrm{d} \tilde{r}\right] .
$$

But, assuming the density is nonincreasing outwards, we have $\rho_{c} \geq \rho(\tilde{r}) \geq \rho(r)$, whence

$$
\zeta(r) \geq \zeta_{c} \exp \left[-\int_{0}^{r} \frac{\mathrm{d} p / \mathrm{d} r}{\rho_{c}+p(\tilde{r})} \mathrm{d} \tilde{r}\right]=\zeta_{c} \frac{\rho_{c}+p_{c}}{\rho_{c}+p(r)} .
$$

Similarly

$$
\zeta(r) \leq \zeta_{c} \exp \left[-\int_{0}^{r} \frac{\mathrm{d} p / \mathrm{d} r}{\rho(r)+p(\tilde{r})} \mathrm{d} \tilde{r}\right]=\zeta_{c} \frac{\rho(r)+p_{c}}{\rho(r)+p(r)} .
$$

We summarize this as a theorem:

Theorem 8 If the density $\rho$ [not the average density] is nonincreasing outwards, then

$$
\zeta(r) \geq \zeta_{c} \frac{\rho_{c}+p_{c}}{\rho_{c}+p(r)} ; \quad \text { and } \quad \zeta(r) \leq \zeta_{c} \frac{\rho(r)+p_{c}}{\rho(r)+p(r)}
$$

If we now evaluate this at the surface of the body:

Corollary 13 If the density $\rho$ [not the average density] is nonincreasing outwards, then

$$
\sqrt{1-2 M / R} \geq \zeta_{c} \frac{\rho_{c}+p_{c}}{\rho_{c}} ; \quad \text { and } \quad \sqrt{1-2 M / R} \leq \zeta_{c} \frac{\rho_{s}+p_{c}}{\rho_{s}} .
$$

A closely related result is obtained by similar bounds applied to the exact general result

$$
\zeta(r)=\sqrt{1-2 M / R} \exp \left[\int_{r}^{R} \frac{\mathrm{d} p / \mathrm{d} r}{\rho(\tilde{r})+p(\tilde{r})} \mathrm{d} \tilde{r}\right] .
$$

Without repeating the details we simply quote the results.

Theorem 9 If the density $\rho$ [not the average density] is nonincreasing outwards, then

$$
\zeta(r) \geq \sqrt{1-2 M / R} \frac{\rho_{s}}{\rho_{s}+p(r)}
$$

and

$$
\zeta(r) \leq \sqrt{1-2 M / R} \frac{\rho(r)}{\rho(r)+p(r)}
$$

These constraints bound the metric components in terms of the pressure and density profiles, and vice versa. A qualitatively different type of constraint is provided by the following result. 
Bounds on the interior geometry and pressure profile of static fluid spheres

Theorem 10 If the sum of the pressure and density is positive

$$
\sqrt{1-2 m(r) / r} \geq \zeta(r) \geq \zeta_{c} \sqrt{1-2 m(r) / r}
$$

Proof: Consider

$$
\begin{aligned}
& \frac{\mathrm{d}}{\mathrm{d} r}\left[\frac{\zeta(r)}{\sqrt{1-2 m(r) / r}}\right]=\frac{\zeta(r)}{\sqrt{1-2 m(r) / r}}\left[g+\frac{[m(r) / r]^{\prime}}{1-2 m(r) / r}\right] . \\
& =\frac{\zeta(r)}{[1-2 m(r) / r]^{3 / 2}}\left[\frac{m+4 \pi p r^{3}}{r^{2}}+\frac{4 \pi \rho r^{2}}{r}-\frac{m}{r^{2}}\right] . \\
& =4 \pi r \frac{\zeta(r)}{[1-2 m(r) / r]^{3 / 2}}[\rho+p] \geq 0 .
\end{aligned}
$$

Therefore

$$
\zeta_{c} \leq \frac{\zeta(r)}{\sqrt{1-2 m(r) / r}} \leq 1
$$

Comment:

Note that this bound makes no reference to the interior Schwarzschild solution for comparison purposes.

Comment:

The condition $\rho+p \geq 0$ is the so-called null energy condition [NEC] - it is certainly satisfied in bulk nuclear matter, though there are exotic situations (typically quantum) in which the NEC is violated [9]. For the purposes of the present article the NEC is automatically assumed in view of our basic hypotheses that pressure and density are both individually positive within the fluid body.

Corollary 14 If the sum of the pressure and density is positive

$$
\zeta_{c}^{2} \leq\left|g_{t t}(r)\right| g_{r r}(r) \leq 1 .
$$

We can now tighten this result by keeping track of the terms we know to be positive a little bit longer. Re-write equation (118) in the form

$$
\frac{\sqrt{1-2 m(r) / r}}{\zeta(r)} \frac{\mathrm{d}}{\mathrm{d} r}\left[\frac{\zeta(r)}{\sqrt{1-2 m(r) / r}}\right]=4 \pi r \frac{\rho+p}{1-2 m(r) / r} .
$$

Whence

$$
\frac{\zeta(r)}{\sqrt{1-2 m(r) / r}}=\exp \left[-\int_{r}^{R} 4 \pi \tilde{r} \frac{\rho(\tilde{r})+p(\tilde{r})}{1-2 m(\tilde{r}) / \tilde{r}} \mathrm{~d} \tilde{r}\right] .
$$

To re-write the RHS, we use the TOV equation written in the form

$$
\frac{\mathrm{d} p}{\mathrm{~d} r}=-\frac{4 \pi r(\rho+p)(\bar{\rho}+3 p)}{3(1-2 m(r) / r)}
$$

to obtain

$$
\frac{\zeta(r)}{\sqrt{1-2 m(r) / r}}=\exp \left[\int_{r}^{R} \frac{3 \mathrm{~d} p / \mathrm{d} r}{\bar{\rho}(\tilde{r})+3 p(\tilde{r})} \mathrm{d} \tilde{r}\right] .
$$


But now if we use our standard assumption that the average density is nonincreasing outwards we have first, $\bar{\rho} \geq \rho_{*}$. Therefore $1 /(\bar{\rho}+3 p) \leq 1 /\left(\rho_{*}+3 p\right)$, and because $\mathrm{d} p / \mathrm{d} r$ is negative, $p^{\prime} /(\bar{\rho}+3 p) \geq p^{\prime} /\left(\rho_{*}+3 p\right)$. Then

$$
\frac{\zeta(r)}{\sqrt{1-2 m(r) / r}} \geq \exp \left[\int_{r}^{R} \frac{3 \mathrm{~d} p / \mathrm{d} r}{\rho_{*}+3 p(\tilde{r})} \mathrm{d} \tilde{r}\right]=\frac{\rho_{*}}{\rho_{*}+3 p(r)} .
$$

Conversely, since $\tilde{r} \in(r, R)$, we have $\bar{\rho}(\tilde{r}) \leq \bar{\rho}(r)$. Therefore $1 /[\bar{\rho}(\tilde{r})+3 p(\tilde{r})] \geq$ $1 /[\bar{\rho}(r)+3 p(\tilde{r})]$, and because $\mathrm{d} p / \mathrm{d} r$ is negative, $p^{\prime} /[\bar{\rho}(\tilde{r})+3 p(\tilde{r})] \leq p^{\prime} /[\bar{\rho}(r)+3 p(\tilde{r})]$. Then performing the integration yields

$$
\frac{\zeta(r)}{\sqrt{1-2 m(r) / r}} \leq \exp \left[\int_{r}^{R} \frac{3 \mathrm{~d} p / \mathrm{d} r}{\bar{\rho}(r)+3 p(\tilde{r})} \mathrm{d} \tilde{r}\right]=\frac{\bar{\rho}(r)}{\bar{\rho}(r)+3 p(r)},
$$

and we have our penultimate theorem:

Theorem 11 If the average density $\bar{\rho}$ is nonincreasing outwards, then

$$
\frac{\zeta(r)\left[\rho_{*}+3 p(r)\right]}{\sqrt{1-2 m(r) / r}} \geq \rho_{*} ; \quad \text { and } \quad \frac{\zeta(r)[\bar{\rho}(r)+3 p(r)]}{\sqrt{1-2 m(r) / r}} \leq \bar{\rho}(r) .
$$

Note that the RHS of the first inequality is independent of location within the gravitating body. Note that this result is definitely more restrictive than equation (80), which was obtained by somewhat simpler techniques. This theorem has several interesting corollaries, easily derived using the comparison bounds on $\zeta(r)$ and $m(r)$. It is easiest to first write

Corollary 15 If the average density $\bar{\rho}$ is nonincreasing outwards, then

$$
\frac{\zeta(r)\left[\rho_{*}+3 p(r)\right]}{\sqrt{1-2 m(r) / r}} \geq \rho_{*}=\frac{\zeta_{*}(r)\left[\rho_{*}+3 p_{*}(r)\right]}{\sqrt{1-2 m_{*}(r) / r}} .
$$

Then, since $\zeta(r) \leq \zeta_{*}(r)$ :

Corollary 16 If the average density $\bar{\rho}$ is nonincreasing outwards, then

$$
\frac{\rho_{*}+3 p(r)}{\sqrt{1-2 m(r) / r}} \geq \frac{\rho_{*}+3 p_{*}(r)}{\sqrt{1-2 m_{*}(r) / r}} .
$$

Similarly, using the fact that $m(r) \geq m_{*}(r)$ :

Corollary 17 If the average density $\bar{\rho}$ is nonincreasing outwards, then

$$
\frac{p(r)}{\sqrt{1-2 m(r) / r}} \geq \frac{p_{*}(r)}{\sqrt{1-2 m_{*}(r) / r}} .
$$

Though the derivation was somewhat complex and lengthy, this last result is by far the cleanest looking bound we have derived on the pressure profile.

Finally, for the central redshift we have:

Corollary 18 If the average density $\bar{\rho}$ is nonincreasing outwards, then

$$
\zeta_{c} \geq \frac{\rho_{*}}{\rho_{*}+3 p_{c}} ; \quad \text { and } \quad \zeta_{c} \leq \frac{\rho_{c}}{\rho_{c}+3 p_{c}} ;
$$


so that

$$
\frac{3 p_{c}}{\rho_{c}} \leq z_{c} \leq \frac{3 p_{c}}{\rho_{*}}
$$

and

$$
\frac{\rho_{*} z_{c}}{3} \leq p_{c} \leq \frac{\rho_{c} z_{c}}{3}
$$

Finally, for our last theorem we start from the exact result

$$
\frac{\zeta(r)}{\sqrt{1-2 m(r) / r}}=\zeta_{c} \exp \left[-\int_{0}^{r} \frac{3 \mathrm{~d} p / \mathrm{d} r}{\bar{\rho}(\tilde{r})+3 p(\tilde{r})} \mathrm{d} \tilde{r}\right],
$$

and again bound $\rho_{c} \geq \bar{\rho}(\tilde{r}) \geq \bar{\rho}(r)$. Without repeating the details is is now easy to see that:

Theorem 12 If the average density $\bar{\rho}$ is nonincreasing outwards, then

$$
\frac{\zeta(r)}{\sqrt{1-2 m(r) / r}} \geq \zeta_{c} \frac{\rho_{c}+3 p_{c}}{\rho_{c}+3 p(r)}
$$

and

$$
\frac{\zeta(r)}{\sqrt{1-2 m(r) / r}} \leq \zeta_{c} \frac{\bar{\rho}(r)+3 p_{c}}{\bar{\rho}(r)+3 p(r)}
$$

\section{Conclusion}

We have extended classic work by Buchdahl [2] and Bondi [3] to derive several modelindependent limits on stellar structure that go beyond what is commonly found in the literature. (Several important papers are those of Kovetz [10], Islam [11], and Forrester [12]; despite the wide variety of inequalities derived in those papers the constraints on the pressure profile derived above seem to be new.) Many of the theorems in this present article are of the form "If the average density is nonincreasing outwards, then generic stellar structure is in many ways bounded by the interior Schwarzschild solution of the same mass and radius". While this is not unexpected, classic results typically refer to global aspects such as the overall compactness $[=2 M / R]$ or the central pressure $p_{c}$ - in this article we have seen how to extend this to local information regarding the metric coefficients and, local compactness, and pressure profile at all points interior to the star. The bounds we derive are sharp in the sense that they are saturated by the interior Schwarzschild geometry, so that in this sense they are the "best possible".

Interest in these issues arose while teaching a course in general relativity and noting that while many textbooks discuss the Buchdahl-Bondi bound, most did not do so by name, and no extant textbook [or, so far as we are aware, the technical literature] covers all the inequalities derived herein.

Further issues that might bear looking at:

- Are there any comparable bounds under more stringent hypotheses?

(For Newtonian stars making the stronger assumption that $\rho$ itself is nonincreasing yields somewhat better bounds on the pressure profile [1]. We have used this hypothesis 
for some of our theorems but suspect that even better bounds may be possible.)

- Are there any comparable nice upper bounds on the pressure?

(We have already seen one upper bound on the pressure profile which unfortunately never gives any information about the central pressure. For some progress on this question see Kovetz [10].)

- Are there similar bounds for solid objects such as planets?

(This leads into the subject of "anisotropic stars" for which some of the best bounds available seem to be those of Guven and O'Murchadha. See [13] and references therein.) - Are there similar bounds for a liquid star enclosed in a solid crust?

(This would require some hybrid techniques - a fluid interior with an anisotropic crust.)

In closing, we reiterate that while the techniques used to derive these bounds are "elementary" in the sense that they can be explained in a introductory course on general relativity, the results are rather non-trivial.

\section{Acknowledgments}

This Research was supported by the Marsden Fund administered by the Royal Society of New Zealand.

\section{References}

[1] S. Chandrasekhar, "Principles of stellar dynamics", (University of Chicago Press, Chicago, 1942).

[2] H. A. Buchdahl, "General Relativistic fluid spheres", Phys. Rev. 116 (1959) 1027-1034; "General Relativistic fluid spheres II: general inequalities for regular spheres", Ap. J. 146 (1966) $275-281$.

[3] H. Bondi, "Massive spheres in general relativity", Mon. Not. Roy. Astron. Soc. 282 (1964) 303-317.

[4] R. M. Wald, "General Relativity", (University of Chicago Press, Chicago, 1984).

[5] S. Weinberg, "Gravitation and cosmology: principles and applications of the general theory of relativity", (Wiley, New York, 1972).

[6] B. K. Harrison, K. S. Thorne, M. Wanako and J. A. Wheeler, "Gravitational theory and gravitational collapse", (University of Chicago Press, Chicago, 1965).

[7] C. W. Misner, K. S. Thorne and J. A. Wheeler, "Gravitation", (W. H. Freeman, San Francisco, 1973).

[8] M. Visser and N. Yunes, "Power laws, scale invariance, and generalized Frobenius series: Applications to Newtonian and TOV stars near criticality", arXiv:gr-qc/0211001.

[9] C. Barcelo and M. Visser, "Twilight for the energy conditions?," Int. J. Mod. Phys. D 11 (2002) 1553 [arXiv:gr-qc/0205066].

[10] A. Kovetz, "Some extremal properties of massive spheres in general relativity", Ap. J. 154 (1968) 241-250;

"Minimal and maximal values of the central pressure and temperature in convectively stable stars", Mon. Not. Roy. Astron. Soc. 144 (1969) 459-460.

[11] J. N. Islam, "Some general relativistic inequalities for a star in in hydrostatic equilibrium", Mon. Not. Roy. Astron. Soc. 145 (1969) 21-29;

"Some general relativistic inequalities for a star in in hydrostatic equilibrium II", Mon. Not. Roy. Astron. Soc. 147 (1970) 377-386.

[12] D. A. Forrester, "Some relativistic integral theorems", Mon. Not. Roy. Astron. Soc. 51 (1970) 149-156. 
[13] J. Guven and N. O'Murchadha, "Bounds on 2m/R for static spherical objects", Phys. Rev. D 60 (1999) 084020 [arXiv:gr-qc/9903067]. 